NOTICE: this is the author's version of a work that was accepted for publication in Planetary and Space Science. Changes resulting from the publishing process, such as peer review, editing, corrections, structural formatting, and other quality control mechanisms may not be reflected in this document. Changes may have been made to this work since it was submitted for publication. A definitive version was subsequently published in Planetary and Space Science, Volume 67, Issue 1, July 2012, pp. 147-154, http://dx.doi.org/10.1016/j.pss.2012.02.006 
Citation: Hirt C, Claessens SJ, Kuhn M, Featherstone WE (2012) Kilometer-resolution gravity field of Mars: MGM2011. Planetary and Space Science, 67(1), 147-154, DOI: 10.1016/j.pss.2012.02.006.

\title{
Kilometer-resolution gravity field of Mars: MGM2011
}

\author{
C. Hirt • S.J. Claessens • M. Kuhn • W.E. Featherstone \\ Western Australian Centre for Geodesy \& The Institute for Geoscience Research, \\ Curtin University of Technology, GPO Box U1987, Perth, WA 6845, Australia \\ Email: c.hirt@curtin.edu.au
}

\begin{abstract}
We present a model that resolves the gravity field of Mars down to km-scales: Mars Gravity Model 2011 (MGM2011). MGM2011 uses Newtonian forward-modelling and the MOLA (Mars Orbiter Laser Altimeter) topography model to estimate the short-scale gravity field (scales of $\sim 3 \mathrm{~km}$ to $\sim 125 \mathrm{~km}$ ). Combined with a reference gravity field and the satellitetracking model MRO110B2, MGM2011 provides surface gravity accelerations and vertical deflections over the entire Martian surface at 3 arc-min resolution. MGM2011 is beneficial for gravity field simulation, inversion and statistics, as well as engineering-driven applications such as topographic mapping and inertial navigation.
\end{abstract}

Keywords: Mars, gravity, topography

\section{Introduction}

Over the past 15 years, international space agencies have placed a strong emphasis on the exploration of Mars, most notably through high-resolution mapping of its topography (e.g., Smith et al., 2001; Gwinner et al., 2010) and observing its external gravity field (e.g., Lemoine et al., 2001; Marty et al., 2010; Konopliv et al., 2011). To date, Mars gravity field determination efforts are commonly based on orbit analysis of artificial Martian satellites. Attenuation of gravity signals at satellite orbit altitude limits the resolution of such models to spatial scales of $\sim 100 \mathrm{~km}$. Opposed to this, the topography of Mars has been accurately measured at a spatial resolution that exceeds that of gravity by $\sim 2-3$ orders of magnitude. Over much of the planet's surface, topography is available at km-scales and finer (e.g., Smith et al., 2001; Gwinner et al., 2010).

The topography is known to be a main contributor of short-wavelength signals to the gravity fields of Earth (e.g., Forsberg and Tscherning, 1981), the Moon (e.g., Goossens et al., 
2011) and the terrestrial planets (e.g., Wieczorek 2007). On Earth, topography models are routinely used in gravity field modelling (Forsberg and Tscherning, 1981; Nagy et al., 2000; Torge, 2001), and gravity field refinement (Pavlis et al., 2007; Hirt et al., 2010). On the Moon, topography data was shown to be a valuable source in estimating the short-scale gravity field (Hirt and Featherstone, 2011).

Here we model the Mars short-wavelength gravity field (in terms of gravity, geoid, vertical deflections) for the first time down to scales of $\sim 3 \mathrm{~km}$ based on the high-resolution MOLA (Mars Orbiter Laser Altimeter) global topography (Smith et al., 2001) and Newtonian forward-modelling (e.g., Nagy et al., 2000). The Newtonian gravity field solution is combined with a reference gravity field and the satellite-derived MRO110B2 model (Konopliv et al., 2011), thus providing a more complete description of the Martian gravity field: Mars Gravity Model 2011 (MGM2011).

\section{Data sets}

We use the global topography model from the MOLA instrument (Smith et al., 2001), operated on the MGS (Mars Global Surveyor) spacecraft between 1998 and 2001. MOLA topography is based on 600 million altimetry ranges extending over most of the Martian surface. The along-track spacing of MOLA altimetry footprints is $0.3 \mathrm{~km}$, with an average across-track spacing of $4 \mathrm{~km}$ at the equator (Smith et al., 2001). The MOLA grid of planetocentric radii of the topography at $1 / 64^{\circ}$ resolution $(\sim 1 \mathrm{~km}$ at the equator) is available through the Planetary Data System (http://pds-geosciences.wustl.edu, product name MGS-MMOLA-5-MEGDR-L3-V1.0). As a spherical harmonic representation of the topography, we use the MarsTopo719 model (Wieczorek, 2007) (http://www.ipgp.fr/ wieczor/SH/SH.html, model MarsTopo719.shape), to reduce the long-wavelength signals from MOLA.

To describe the long-wavelength gravity field of Mars, the 110-degree spherical harmonic potential model MRO110B2 (Konopliv et al., 2011) is chosen. MRO110B2 is the latest Martian gravity field from the Jet Propulsion Laboratory, based on 8.5 years of MGS and 6 years of Odyssey Doppler and range tracking data. MRO110B2 also incorporates 2 years of low-orbiting Mars Reconnaissance Orbiter (MRO) tracking data, which is why its spatial resolution is an improvement over earlier models (Konopliv et al., 2011). MRO110B2 is available as a set of fully-normalised coefficients via (http://pds-geosciences.wustl.edu, product name jgmro_110b2_sha.tab). 


\section{MGM2011 Development}

MGM2011 is composed of three constituents: First, a reference gravity field is introduced to take into account the height-dependent gravitational attraction of Mars, as approximated by a rotating mass-ellipsoid of homogeneous density. Second, the gravitational potential model MRO110B2 serves as a source for medium- to long-wavelength Mars gravity field structures. Third, beyond the resolution of the potential model, Newtonian forward-modelling estimates the fine structure of the gravity field, as implied by the high-resolution MOLA topography. This approach parallels the successful construction of surface gravity accelerations on Earth (Hirt et al., 2011a) and the Moon (Hirt and Featherstone, 2011). All MGM2011 constituents are computed in terms of $1 / 20^{\circ}$ global grids ( $\sim 3 \mathrm{~km}$ resolution at the equator).

\subsection{Reference gravity field}

The concept of the equipotential ellipsoid, an integral part of the Geodetic Reference System 1980 (Moritz, 1980) used in Earth geodesy, is adopted here to provide the reference for MGM2011. By convention, we use the following set of four defining parameters, the semimajor axis $a$, semi-minor axis $b$, the product of the gravitational constant and the planetary mass $G M$ and the angular velocity of planetary rotation $\omega$. These define the geometry and gravitational attraction of a bi-axial equipotential ellipsoid of Somigliana-Pizzetti type (Moritz, 1980; Torge, 2001, chapter 4). To establish the Mars Geodetic Reference System (MGRS), we use the numerical values for $a, b, G M$ and $\omega$ (Table 1), as published by Ardalan et al. (2010). Their $a$ and $b$-values provide a best fit between the Mars geoid and ellipsoid, in that, they minimize the geoid-ellipsoidal separation in a least-squares sense and keep other Mars gravity field functionals (e.g., gravity disturbances, vertical deflections) small.

Following Moritz's formalism given in Appendix A1, the MGRS geometric and physical constants were derived from the four defining parameters. The MGRS defining and derived constants (Table 1) are used for the computation of the normal gravity (Appendix A2) at the topography of Mars, as represented by the MOLA-3D locations. Because the normal gravity field accounts for the ellipticity of Mars, we subtract the MGRS zonal harmonics (to order 10, Table 1) from the corresponding coefficients of the MRO110B2 potential model (Appendix A3).

We acknowledge other numerical values to define a MGRS are available in the literature, e.g., the ellipsoid parameters of the International Astronomical Union (IAU), $a^{\mathrm{IAU}}$ $=3396190 \mathrm{~m}$ and $b^{\mathrm{IAU}}=3376200 \mathrm{~m}$ (cf. Seidelmann et al., 2002, p. 103) that define a bestfitting ellipsoid with respect to the Mars topography. Here we prefer the Ardalan et al. (2010) parameters (Table 1) over the IAU constants because they keep MGM2011 gravity field 
quantities (geoid, gravity disturbances, and vertical deflections) smaller. Computation of alternative Mars reference gravity fields, e.g., based on the IAU ellipsoid parameters, is straightforward with the equations given in the Appendix.

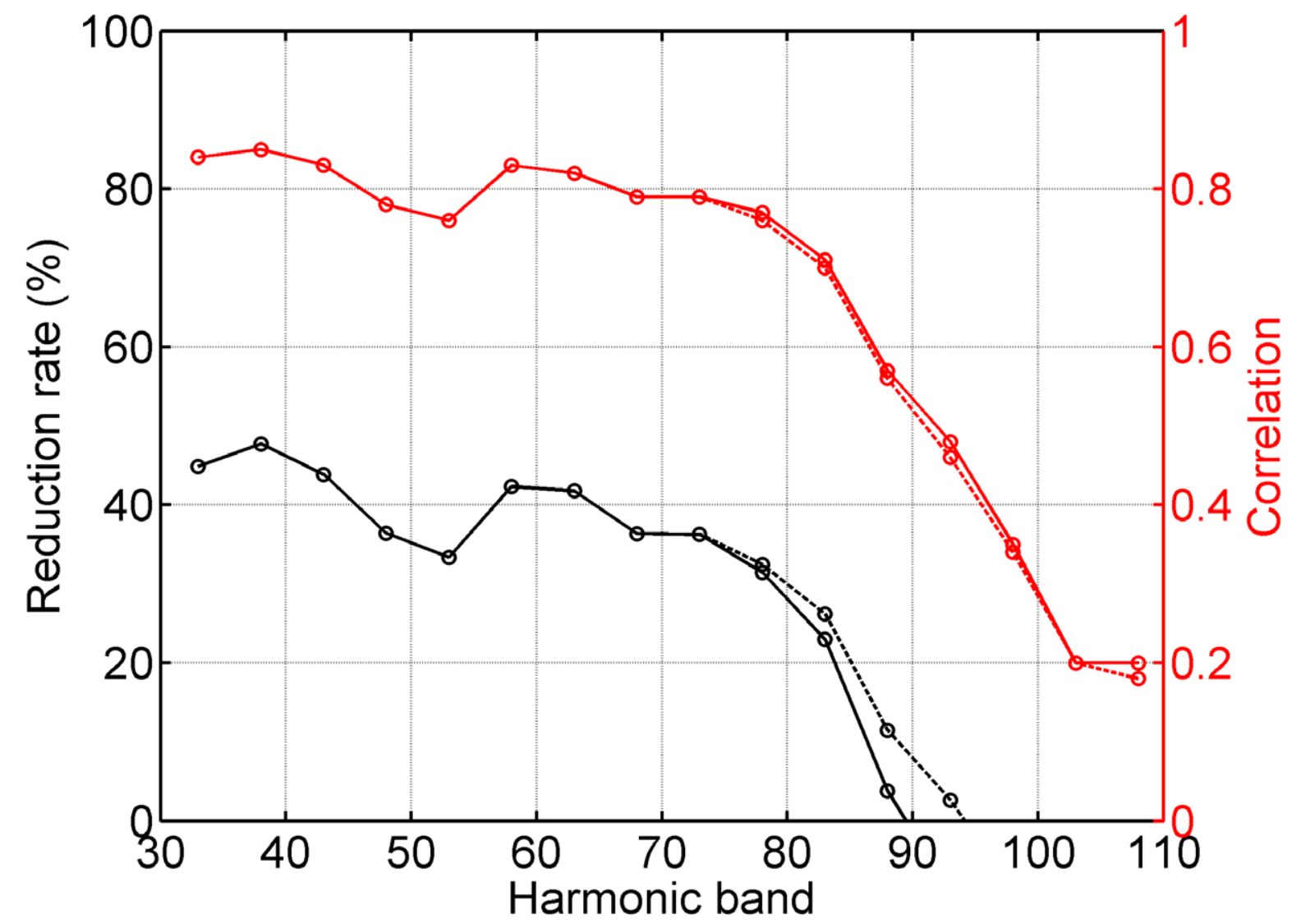

Fig. 1. Reduction rates (black) and cross-correlation coefficients (red) between topographyimplied gravity $g_{\text {TOP }}$ and MRO110B (solid) and MRO110B2 (dotted) gravity $g_{M R O}$. Reduction rates computed from $\left.100 \% \cdot\left[R M S\left(g_{T O P}\right)-R M S\left(g_{T O P}-g_{M R O}\right)\right] / R M S\left(g_{T O P}\right)\right)$.

\subsection{Potential model}

To initially assess the short-scale quality of MRO110B2, and of MRO110B (a constraint variant published by Konopliv et al., (2011)), we have computed band-limited gravity from the MarsTopo719 topography model. Global 1/4º-resolution grids of MarsTopo719 were created for a range of 5-degree wide spectral bands (e.g., harmonic degrees 31 to 35), converted to gravity $g_{\text {TOP }}$ using Newtonian forward-modelling (see Section 3.3 and Pavlis et al. 2007) and compared with MRO110B2 gravity $g_{M R O}$ at the same locations and spectral bands over the entire surface of Mars. To analyse the (global) agreement between $g_{\text {TOP }}$ and $g_{M R O}$, we use reduction rates (RRs), see Fig. 1 . RRs quantify the extent the RMS (root mean 
square) signal strength of $g_{\text {TOP }}$ is reduced ('explained') by the MRO110B gravity $g_{M R O}$. RRs are most sensitive to indicate the strength of topography-generated gravity signals $g_{\text {TOP }}$ captured by potential models.

For each 5-degree spectral band, Fig. 1 shows the correlation between $g_{T O P}$ and $g_{M R O}$, and RRs, which are at the level of $\sim 40 \%$ to harmonic degree 70 and drop below $20 \%$ beyond degree 85, showing that topography signals are not sufficiently captured anymore. As input model for MGM2011, we chose MRO110B2 because it better represents topography signals than the more constrained MRO110B (Fig. 1). The attenuated MRO110B2 information beyond degree 85 is not used. MRO110B2 gravity field functionals (gravity disturbances and vertical deflections) were evaluated in spectral band 2 to 85 at the 3D locations (latitude, longitude, planetocentric radii) of the MOLA topography (see Appendix A3) using an adaption of the harmonic_synth software (Holmes and Pavlis, 2008)

\subsection{Newtonian forward-modelling}

The spherical harmonic topography, MarsTopo719, expanded to degree 85, was subtracted from the MOLA $1 / 64^{\circ}$ elevation model, yielding a residual terrain model (RTM, Forsberg, 1984). The MarsTopo719 model thus serves as a spectral filter (e.g., Hirt, 2010) to reduce those signals from MOLA that are delivered by MRO110B2. The RTM represents a grid of mass-prisms, providing rich high-frequency information on the expected Martian gravity field at scales shorter than $\sim 125 \mathrm{~km}$. Newtonian forward-modelling (Forsberg, 1984; Nagy et al., 2000; Pavlis et al., 2007; Hirt, 2010; Hirt et al., 2010) was applied to convert the Mars RTM elevations to 3 arc min grids of gravity disturbances, geoid and vertical deflections, see Appendix A4. We denote the forward-modelled gravity field functionals MRTM85 (Mars RTM with the spectrum to degree 85 removed).

The forward-modelling procedure was carried out through numerical integration with a variant of the TC software (Forsberg, 1984) using a uniform mass-density of $\sim 2900 \mathrm{~kg} \mathrm{~m}^{-3}$ for the topography. This is the average value of mass-densities of Martian crust ranging between 2700 to $3100 \mathrm{~kg} \mathrm{~m}^{-3}$ used by Wieczorek and Zuber (2004). MRTM85 functionals are only estimates of the high-frequency Martian gravity field, and based on the assumptions of uncompensated and constant-mass density topography. Given that the (uncompensated) topography is thought to be the dominant source of short-scale planetary gravity fields (Wieczorek, 2007; Hirt et al., 2010; Goossens et al., 2011; Hirt and Featherstone, 2011) we infer that MRTM85 is a reasonable approximation of the short-scale Martian gravity field (see also Sect. 5) within the following limitations. 
Long-wavelength topographic features of Mars's topography were found to show isostatic compensation (e.g., Neumann et al., 2004, Fig. 6 ibid). For Earth, Torge (2001, p340), states that topographic "loads of several 10 to $100 \mathrm{~km}$ dimensions are supported by the strength of the lithosphere and are not isostatically compensated”, suggesting that the shorter the scales of topographic features, the better will be the support through the crust (see also Watts 2001, p. 176). In light of the $40-50 \mathrm{~km}$ strong Martian crust (e.g., Wieczorek, 2007) we conclude that the crustal support of Mars's topography will be increasingly better the shorter the spatial scales while there is scope for compensation effects at longer spatial scales, say $100 \mathrm{~km}$, which are not modelled by MRTM85.

Our constant mass-density assumption of $2900 \mathrm{~kg} \mathrm{~m}^{-3}$ is a simplification of the actual mass-density distribution of Mars's lithosphere. Based on spectral analyses of Martian topography and gravity, McGovern et al. (2002) estimated mass-densities for individual regions of Mars. They found a best-fitting value of $2900 \mathrm{~kg} \mathrm{~m}^{-3}$ (used here for MRTM85) for the transfer function between topography and gravity (aka admittance) over several regions of Mars, see also Neumann et al. (2004). McGovern et al. (2002) suggests mass-densities of up to $\sim 3150 \mathrm{~kg} \mathrm{~m}^{-3}$ over the Tharsis Montes region, and values possibly as low as $\sim 2000 \mathrm{~kg} \mathrm{~m}^{-3}$ over the Northern parts of Valles Marineris. Regional mass-density variations, as suggested by e.g., McGovern et al. (2002) and Neumann et al. (2004) are not taken into account here because we do not have accurate maps of their locations, depths and extents to hand. We believe that our forward-modelled gravity effects will agree reasonably well with the real Martian gravity field where the actual mass-density is close to our assumption. Conversely, larger discrepancies will be present where the actual deviates strongly from our assumed mass-density. A refined forward-modelling that incorporates regionally varying massdensities, and also the polar ice caps, remains for a future task should these 3D models become available to us.

\section{Results}

\subsection{Gravity acceleration}

The variation of MGM2011 gravity accelerations over the entire Martian surface is shown in Fig. 2a. Gravity accelerations are the sum of the three input components MGRS normal field (Fig. 2b), MRO110B2 gravity disturbances in spectral band 2 to 85 (Fig. 2c) and MRTM85 Newtonian gravity (Fig. 2d). MGM2011 gravity disturbances (Fig. 3) are the sum of MRO110B2 and MRTM85 gravity. From Table 2, the average surface gravity acceleration is $3.72076 \mathrm{~m} / \mathrm{s}^{2}$ with a global variation range of $\sim 0.059 \mathrm{~m} / \mathrm{s}^{2}$ or $1.6 \%$. MGM2011 gravity 
accelerations are maximum at the bottom the Jojutla crater $\left(81.6^{\circ} \mathrm{N}, 169.3^{\circ} \mathrm{W}\right)$ in the lowlying Northern plains and minimum at the rim of Arsia Mons $\left(8.4^{\circ} \mathrm{S}, 121.4^{\circ} \mathrm{W}\right)$, the southernmost of the Tharsis shield volcanos.
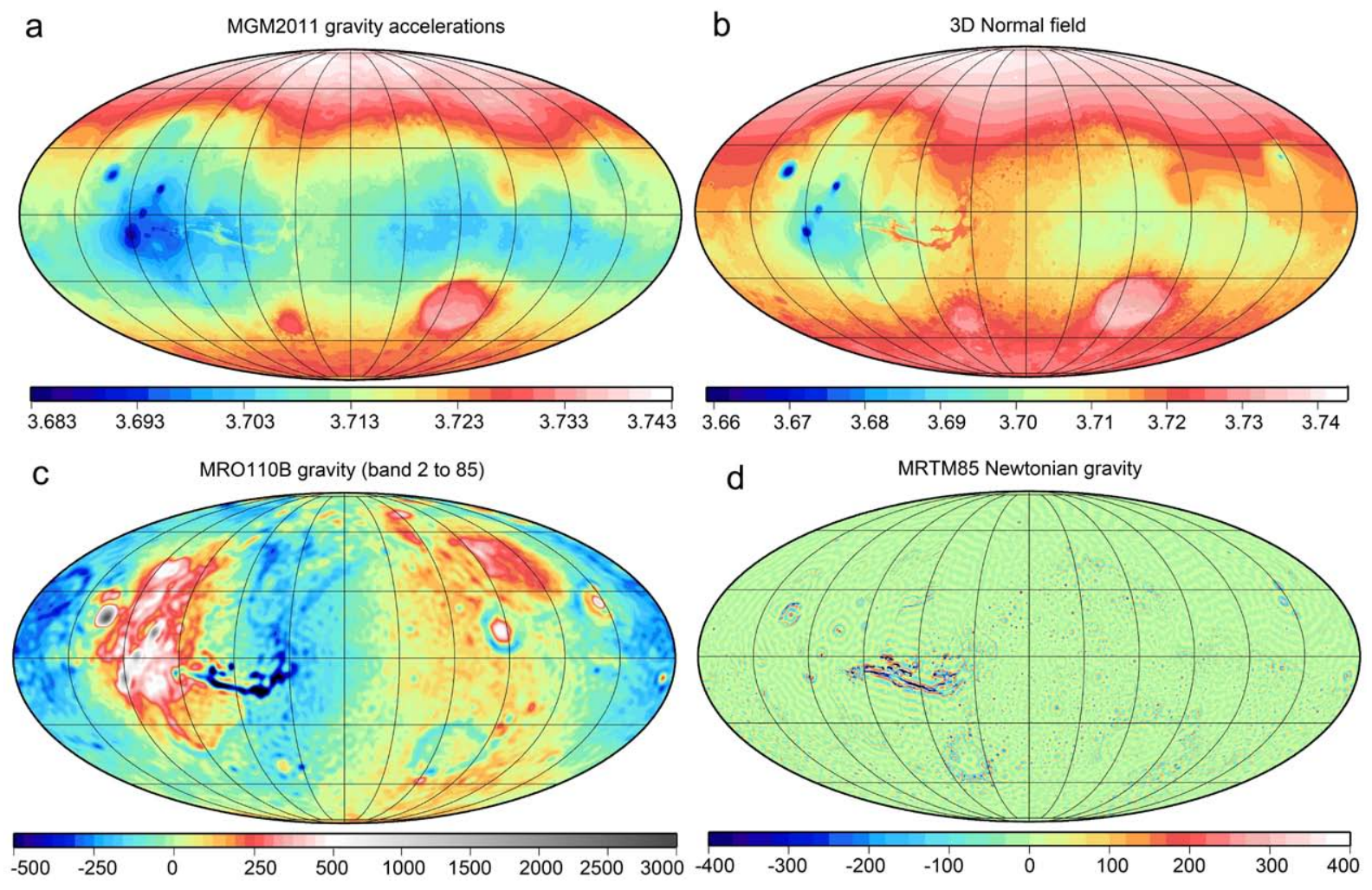

Fig. 2. (a) MGM2011 surface gravity accelerations, (b) MGM2011 3D-normal gravity field, evaluated at the elevations of the topography above the MGRS ellipsoid surface, (c) MRO110B gravity disturbances (spectral band 2 to 85, evaluated at the radii of the topopgraphy), (d) MRTM85 gravity, Units are $\mathrm{m} \mathrm{s}^{-2}$ (panels a, b) and mGal (panels c, d). Mollweide projection centred at $0^{\circ}$ longitude.

From Fig. 2d, Newtonian forward-modelling resolves the expected gravity field signatures of impact craters and other topographic features at spatial scales shorter than 125 km. This is also seen from a comparison between Figs. 2c and 3. MRTM85 amplitudes are often at the $100 \mathrm{mGal}-$ level. Over the entire planet, the MRTM85 gravity disturbance strength is $\sim 41 \mathrm{mGal}$ (Table 2). Lemoine et al. (2001, p. 23370) estimated $\sim 9 \mathrm{mGal}$ RMS for Martian gravity signals beyond harmonic degree 60. However, the MRO11B2 RMS gravity signal is $\sim 23 \mathrm{mGal}$ in band 61 to 85 . Our results suggest that short-scale gravity signals (beyond degree 60) were underestimated by a factor $\sim 5$ in Lemoine et al. (2001) in the past.

MGM2011 gravity can be used for Mars gravity field simulation, as required for the design of future high-resolution Mars satellite gravity mapping mission (e.g., gradiometry). 
MGM2011 can be used as an a priori model for inversion of present and future spacecraft gravity data, for future landing missions, e.g., prediction of surface gravity and the local vertical at prospective landing sites, and Mars gravity field statistics. Given that MRTM85 relies on the assumptions of uncompensated and constant mass-density topography, MGM2011 cannot be used for direct geological interpretation.

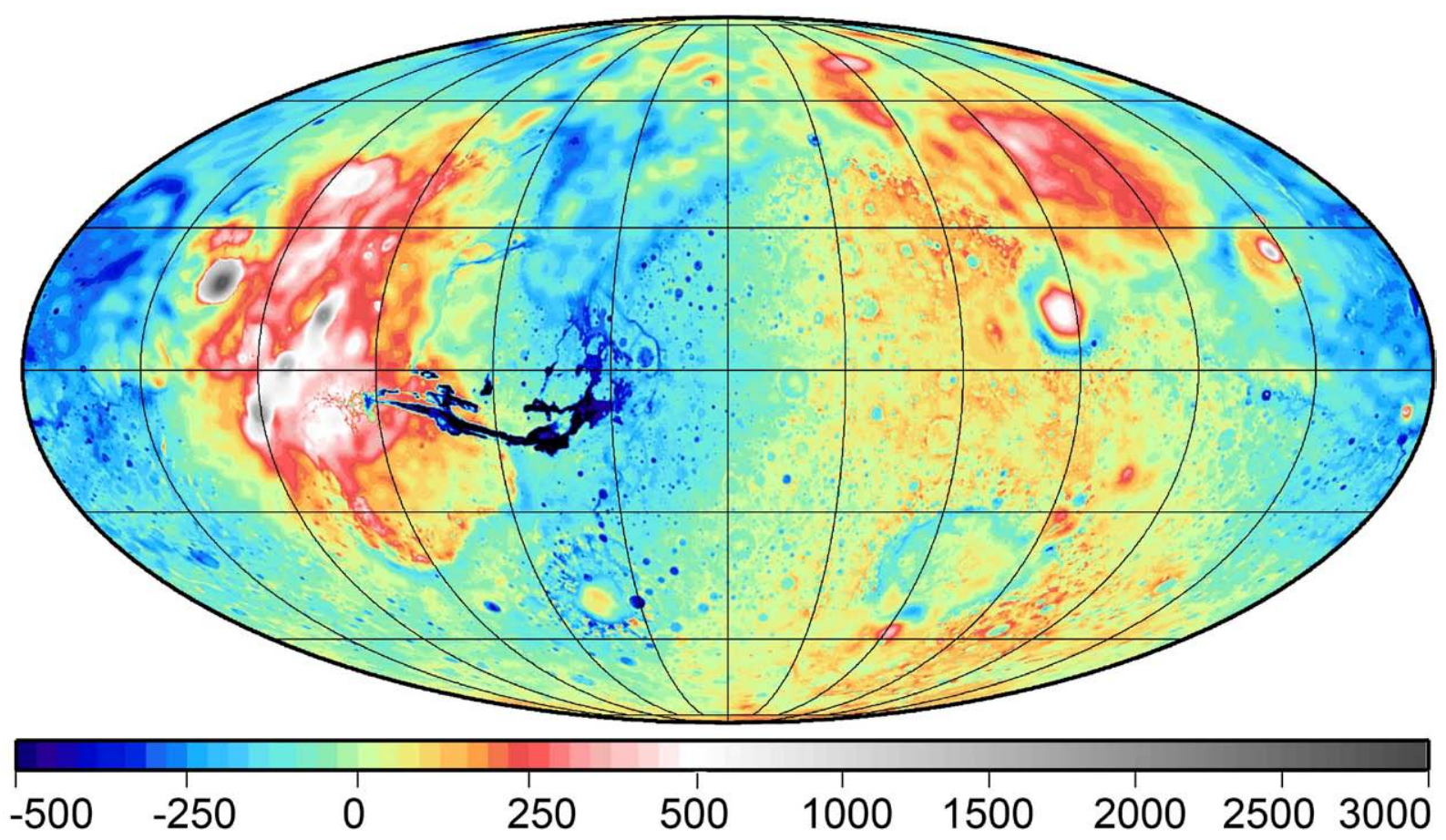

Fig. 3. MGM2011 free-air gravity disturbances (in $\mathrm{mGal}$ ), Mollweide projection centred at $0^{\circ}$ longitude

\subsection{By-products}

MGM2011 by-products are MRTM85 geoid undulations and vertical deflections (Table 3). The MRTM85 geoid undulations exhibit signals of $2.3 \mathrm{~m}$ RMS with amplitudes of some $10 \mathrm{~m}$ in places. MRTM85 can be added to MRO110B2 or any other Mars spherical harmonic model for geoid refinement beyond harmonic degree 85. Applications might be in highresolution topographic mapping (Gwinner et al., 2010), where the geoid takes the role of a height reference surface, specifically in local analyses of gravity-driven mass movements.

MGM2011 vertical deflections, the sum of MRO110B2 and MRTM85 vertical deflections apply to the surface of Mars and are provided in terms of North-South and EastWest components with respect to the projected surface normal of MGRS (Table 3). The magnitude of the MGM2011 total vertical deflections over the entire Martian surface is shown in Fig. 4. Maximum values in excess of 1000 arc seconds are found near $18^{\circ} \mathrm{N}, 136^{\circ} \mathrm{W}$, West 
of Olympus Mons. The knowledge of vertical deflections, as delivered by MGM2011, may be important for future precision inertial navigation at or over Mars (cf Grejner-Brzezinska and Wang, 1998; Jekeli, 2001).

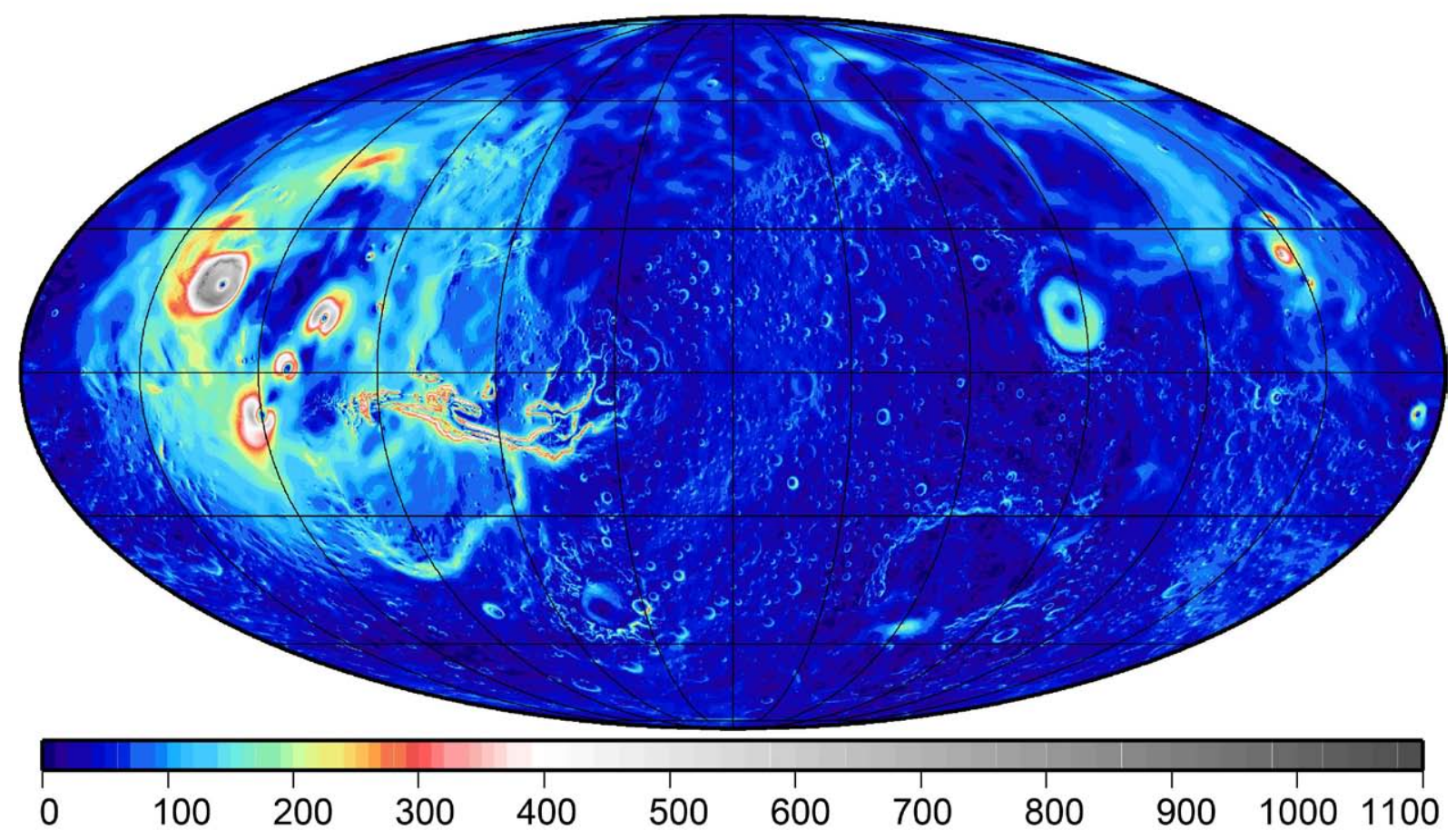

Fig. 4. MGM2011 total vertical deflections $\left(\sqrt{N S D o V^{2}+E W D o V^{2}}\right)$ in arc seconds. Mollweide projection centred at $0^{\circ}$ longitude.

\section{Model assessment}

The MGM2011 construction is validated implicitly because it is based on the same tried and tested principles used successfully in the construction of surface gravity accelerations on Earth (Hirt et al., 2011) and the Moon (Hirt and Featherstone, 2011). The comparison between $g_{\text {TOP }}$ and $g_{M R O}$ (Fig. 1) provides a check on the Newtonian forward-modelling procedure used to create MRTM85. Direct MGM2011 validation with ground-truth observations is not possible. To indirectly evaluate MGM2011, we have performed an asclose-as-possible replication on Earth as planet with dense ground-truth data sets available (Hirt et al., 2011b). The replication uses exactly the same modelling approach with identical assumptions and most similar parameters, and, importantly, uses topography-implied gravity at scales of $\sim 125$ to $\sim 3 \mathrm{~km}$ to augment satellite-only gravity.

Comparison of the MGM2011-replication with six dense ground-truth data sets (gravity, geoid, vertical deflections) over Europe and North America show that the MGM2011 modelling approach improves satellite-only gravity models in rugged terrain. The 
improvement is most significant for gravity (55\% to 67\%), substantial for vertical deflections (30\% to $50 \%$ ) and notable for geoid undulations ( 12\% and $47 \%$ ). As a limitation of the short-scale modelling technique, information on isostatic compensation or local mass-density anomalies are not conferred by the residual topography, while those signals originating from the uncompensated topography are modelled reasonably well over regions where the actual mass-density is close to our constant density value. Because the correlation between gravity and topography is higher for Mars than Earth at 100-km-scales (e.g., Wieczorek, 2007; Hirt et al., 2011b), it is reasonable to conclude that the MGM2011-approach is effective at approximating Mars's short-scale gravity field, specifically over the rugged southern hemisphere.

In order to assess the impact of the MGRS defining parameters $a$ and $b$ on the MGM2011 surface gravity accelerations we have repeated the MGM2011 development in its entirety based on the IAU Mars ellipsoid parameters $a^{\mathrm{IAU}}$ and $b^{\mathrm{IAU}}$ (see Section 3.1). Comparison of this alternative solution with MGM2011 gravity accelerations (based on the MGRS parameters in Table 1) showed a RMS agreement smaller than 1 mgal. Given that the MGRS and IAU ellipsoid parameters differ by $760 \mathrm{~m}$ (major axis a) and 1500 m (major axis b), the choice of the reference system parameters $a$ and $b$ has no significant impact on the constructed surface gravity.

\section{Concluding remarks}

MGM2011 is the spectrally most complete estimation of the external Martian gravity field to date. Forward-modelling was used to estimate the expected high-frequency gravity field. MGM2011 can be applied in Mars gravity field modelling (background model for the inversion of spacecraft data, simulation studies for future high-resolution satellite gravity field missions and statistical analysis), though it is not recommended for direct geological interpretation. MGM2011 is beneficial for engineering-driven applications (mapping, landing spacecraft and near-surface inertial navigation), which are the foundation of geophysical data acquisition. The MGM2011 model (including input data sets) is freely available at www.geodesy.curtin.edu.au/research/models/mgm2011.

Acknowledgements We thank the Australian research council for funding through Discovery Project Grant DP0663020. 


\section{ppendix}

\section{A1 Computation of the MGRS reference gravity field}

Four defining constants [ $a$ (semi-major axis of the reference ellipsoid), $b$ (semi-minor axis), GM (product of the gravitational constant and the planetary mass) and $\omega$ (angular velocity of the planetary rotation)] are used to derive the MGRS geometric and physical constants based on the formulas in Moritz (1980, 2000). The geometric flattening is

$$
f=\frac{a-b}{a}
$$

the first numerical eccentricity is

$$
e=\frac{\sqrt{a^{2}-b^{2}}}{a}
$$

and the second numerical eccentricity is

$e^{\prime}=\frac{\sqrt{a^{2}-b^{2}}}{b}$

The physical constants are the ratio of the centrifugal acceleration to normal gravity at the planetary equator

$m=\frac{\omega^{2} a^{2} b}{G M}$

and the dynamic form factor

$$
J_{2}=\frac{e^{2}}{3}\left(1-\frac{2 m e^{\prime}}{15 q_{0}}\right)
$$

where

$$
q_{0}=\frac{1}{2}\left[\left(1+\frac{3}{e^{\prime 2}}\right) \arctan e^{\prime}-\frac{3}{e^{\prime}}\right]
$$

is an auxiliary parameter. The other even zonal harmonics $J_{4}$ to $J_{10}$ of the reference ellipsoid are computed recursively from

$$
J_{2 n}=(-1)^{n+1} \frac{3 e^{2 n}}{(2 n+1)(2 n+3)}\left(1-n+5 n \frac{J_{2}}{e^{2}}\right)
$$

where $n$ is the spherical harmonic degree. The un-normalized $J_{2 n}$-coefficients are converted to fully-normalized coefficients by

$$
\bar{C}_{2 n}=\frac{1}{\sqrt{4 n+1}} J_{2 n}
$$

Finally, another auxiliary parameter 


$$
q_{0}^{*}=3\left(1+\frac{1}{e^{\prime 2}}\right)\left(1-\frac{1}{e^{\prime}} \arctan e^{\prime}\right)-1
$$

is required to compute normal gravity at the equator

$$
\gamma_{a}=\frac{G M}{a b}\left(1-m-\frac{m}{6} e^{\prime} \frac{q_{0}^{*}}{q_{0}}\right)
$$

and normal gravity at the poles

$$
\gamma_{b}=\frac{G M}{a^{2}}\left(1+\frac{m}{3} e^{\prime} \frac{q_{0}^{*}}{q_{0}}\right)
$$

of the reference ellipsoid. Numerical values for the MGRS defining and derived constants are listed in Table 1. Computation of alternative reference gravity fields, e.g., based on the IAU ellipsoid parameters (Seidelmann et al., 2002), is straightforward with the above equations.

\section{A2 Computation of normal gravity at 3D-locations}

The spherical coordinates, comprising $\varphi$ (planetocentric latitude), $\lambda$ (planetocentric longitude) and $r$ (planetocentric radius) describe the 3D-locations of Mars's topography, as represented by MOLA. The planetodetic (geodetic) latitude $\phi$, required to compute normal gravity at MOLA 3D-locations is obtained through (e.g., Torge 2001, p 95)

$$
\phi \approx \arctan \left(\frac{\tan \varphi}{1-e^{2}}\right)
$$

Normal gravity $\gamma_{0}(\phi)$ at the surface of the MGRS ellipsoid at $\phi$ is computed from (Moritz 2000, p 132)

$$
\gamma_{0}(\phi)=\gamma_{a} \frac{1+k \sin ^{2} \phi}{\left(1-e^{2} \sin ^{2} \phi\right)^{0.5}}
$$

where

$$
k=\frac{b \gamma_{b}}{a \gamma_{a}}-1
$$

To compute normal gravity at the MOLA 3D-locations $(\varphi, \lambda, r)$, a second-order series expansion is applied (e.g., Torge 2001 p. 110)

$$
\gamma(h, \phi) \approx \gamma_{0}(\phi)\left(1-\frac{2}{a}\left(1+f+m-2 f \sin ^{2} \phi\right) h+\frac{3}{a^{2}} h^{2}\right)
$$

which is independent of the planetocentric longitude $\lambda$. The ellipsoidal heights $h$ of the MOLA topography related to the MGRS ellipsoid are computed by subtracting the MGRS ellipsoidal radii $r_{e}(\varphi)$ 
$r_{e}(\varphi)=a \sqrt{\frac{1-e^{2}}{1-e^{2} \sin ^{2} \varphi}}$

(cf. Claessens 2006, p19) from the planetocentric radii of the MOLA 3D-locations:

$h \approx r-r_{e}(\varphi)$.

\section{A3 Potential model evaluation}

Let $M$ be the maximum spherical harmonic degree, $G M^{M R O}$ and $a^{M R O}$ the MRO110B2 modelspecific constants, and $\bar{C}_{n m}^{M R O} \bar{S}_{n m}^{M R O}$ the fully-normalized MRO110B2 spherical harmonic coefficients of degree $n$ and order $m$. Because the MRO110B2 model-specific constants $\left(G M^{M R O}=4.2828374526 \times 10^{13} \mathrm{~m}^{3} \mathrm{~s}^{-2}\right.$ and $\left.a^{M R O}=3396000 \mathrm{~m}\right)$ differ from the respective $G M$ and $a$ values of the MGRS (cf. Table 1), the coefficient transformation

$$
\delta \bar{C}_{n m}^{M R O}=\bar{C}_{n m}^{M R O}-\frac{G M}{G M^{M R O}} \cdot\left(\frac{a}{a^{M R O}}\right)^{n} \cdot \bar{C}_{n m}
$$

(e.g., Smith 1998, Eq. 5 ibid) was used to make the zonal harmonics $\bar{C}_{n m}$ of the MGRS compatible with those of the MRO110B2 potential model. The term $\delta \bar{C}_{n m}$ denotes the MRO110B2 zonal harmonic coefficients with the zonal harmonics of the MGRS removed. Equation (A18) is evaluated for the first five even zonal harmonics $\bar{C}_{2,0}$ to $\bar{C}_{10,0}$ of the MGRS.

The spherical harmonic series expansions used to evaluate MRO110B2 functionals were derived from the disturbing potential $T$ (Torge 2001, Eq. 6.4)

$$
T(\varphi, \lambda, r)=\frac{G M}{r} \sum_{n=2}^{M}\left(\frac{a}{r}\right)^{n} \sum_{m=0}^{n}\left(\overline{\delta C}_{n m}^{M R O} \cos m \lambda+\bar{S}_{n m}^{M R O} \sin m \lambda\right) \bar{P}_{n m}(\sin \varphi)
$$

yielding gravity disturbances $\delta g$, North-South $\xi$ and East-West $\eta$ vertical deflections:

$$
\begin{aligned}
& \delta g^{M R O}(\varphi, \lambda, r)=-\frac{\partial T}{\partial r}=\frac{G M}{r^{2}} \sum_{n=2}^{M}(n+1)\left(\frac{a}{r}\right)^{n} \sum_{m=0}^{n}\left(\overline{\delta C}_{n m}^{M R O} \cos m \lambda+\bar{S}_{n m}^{M R O} \sin m \lambda\right) \bar{P}_{n m}(\sin \varphi) \\
& \xi^{M R O}(\varphi, \lambda, r)=-\frac{1}{\gamma r} \frac{\partial T}{\partial \varphi}=-\frac{G M}{\gamma r^{2}} \sum_{n=2}^{M}\left(\frac{a}{r}\right)^{n} \sum_{m=0}^{n}\left(\overline{\delta C}_{n m}^{M R O} \cos m \lambda+\bar{S}_{n m}^{M R O} \sin m \lambda\right) \bar{P}_{n m}^{\prime}(\sin \varphi)
\end{aligned}
$$


$\eta^{M R O}(\varphi, \lambda, r)=-\frac{1}{\gamma r \cos \varphi} \frac{\partial T}{\partial \lambda}=-\frac{G M}{\gamma r^{2} \cos \varphi} \sum_{n=2}^{M}\left(\frac{a}{r}\right)^{n} \sum_{m=0}^{n} m\left(\bar{S}_{n m}^{M R O} \cos m \lambda-\overline{\delta C}_{n m}^{M R O} \sin m \lambda\right) \bar{P}_{n m}(\sin \varphi)$

where $\bar{P}_{n m}(\sin \varphi)$ are the fully-normalized associated Legendre functions of degree $n$, order $m$, and $\bar{P}_{n m}^{\prime}(\sin \varphi)$ is the first derivative of $\bar{P}_{n m}(\sin \varphi)$. The series expansions in Eqs. (A19 to A22) were evaluated with the MRO110B2 coefficients to $M=85$ at Mars's surface, as represented through the 3D-MOLA topography $(\varphi, \lambda, r)$.

\section{A4 Newtonian forward-modelling}

Newtonian forward-modelling was applied to convert the MOLA residual topography to topography-implied gravity field functionals. The required MOLA residual terrain model (MRTM) was constructed by subtracting a harmonic reference surface from the MOLA planetocentric radii $r(\varphi, \lambda)$ :

$$
z^{R T M}(\varphi, \lambda)=r(\varphi, \lambda)-\sum_{n=0}^{M} \sum_{m=0}^{n}\left(\overline{H C}_{n m} \cos m \lambda+\overline{H S}_{n m} \sin m \lambda\right) \bar{P}_{n m}(\cos \varphi)
$$

where the $\overline{H C}_{n m}$ and $\overline{H S}_{n m}$ are the coefficients of the spherical harmonic reference surface MarsTopo719 to $M=85$. Our Newtonian forward-modelling procedure treats $Z^{\text {MRTM }}$ as heights of right-rectangular mass-prisms being 'building blocks' of the residual topography (Forsberg, 1984). The gravitational potential of the prisms is evaluated using analytical closed-form expressions, and the effects of all mass-elements within some radius around the computation point are superposed to obtain the gravity effects implied by the entire residual topography (below).

The equations used to convert the $z^{\text {MRTM }}$ elevations to gravity field functionals: geoid $N^{\text {MRTM }}$, gravity disturbances $\delta g^{\text {MRTM }}$, vertical deflections $\xi^{\text {MRTM }}$ (North-South component), $\eta^{R T M}$ (East-West component) read in planar approximation (after Forsberg, 1984; Nagy et al., 2000; Hirt, 2010 and Hirt et al., 2010)

$$
\begin{aligned}
N^{M R T M}= & \sum_{1}^{k} \frac{G \rho}{\gamma} \mid \| x z \ln (z+c)+y z \ln (x+c)+z x \ln (y+c) \\
& -\frac{x^{2}}{2} \tan ^{-1} \frac{y z}{x c}-\frac{y^{2}}{2} \tan ^{-1} \frac{z x}{y c}-\left.\left.\left.\frac{z^{2}}{2} \tan ^{-1} \frac{x y}{z c}\right|_{x_{1}} ^{x_{2}}\right|_{y_{1}} ^{y_{2}}\right|_{z_{1}} ^{z_{2}} \\
\delta g^{M R T M}= & h c-\left.\left.\sum_{1}^{k} G \rho\left|\| x \ln (y+c)+y \ln (x+c)-z \tan ^{-1} \frac{x y}{x c}\right|_{x_{1}}^{x_{2}}\right|_{y_{1}} ^{y_{2}}\right|_{z_{1}} ^{z_{2}},
\end{aligned}
$$




$$
\begin{aligned}
& \xi^{M R T M}=-\frac{1}{\gamma} \sum_{1}^{k} G \rho \|\left.\left.\left|y \ln (z+c)+z \ln (y+c)-x \tan ^{-1} \frac{y z}{x C}\right|_{x_{1}}^{x_{2}}\right|_{y_{1}} ^{y_{2}}\right|_{z_{1}} ^{z_{2}}, \\
& \eta^{M R T M}=-\frac{1}{\gamma} \sum_{1}^{k} G \rho \|\left.\left.\left|z \ln (x+c)+x \ln (z+c)-y \tan ^{-1} \frac{x z}{y c}\right|_{x_{1}}^{x_{2}}\right|_{y_{1}} ^{y_{2}}\right|_{z_{1}} ^{z_{2}} .
\end{aligned}
$$

where $\mathrm{G} \approx 6.673 \times 10^{-11} \mathrm{~m}^{3} \mathrm{~kg}^{-1} \mathrm{~s}^{-2}$ denotes the Universal Gravitational constant, $\rho$ the massdensity of the residual topography (here $2900 \mathrm{~kg} \mathrm{~m}^{-3}$ ) and $\gamma \approx 3.72 \mathrm{~m} \mathrm{~s}^{-2}$ mean gravity acceleration of Mars, $(x, y, z)$ are planar coordinates, $c$ the distance between the point $(x, y, z)$ from the origin of the coordinate system $(0,0,0)$ (cf. Nagy et al., 2000). The variables $(x, y, z)$ are substituted by the corner coordinates of the prism $\left(x_{1}, y_{1}, z_{1}, x_{2}, y_{2}, z_{2}\right)$. We use $z_{1}=0$ and $z_{2}=z^{R T M}$, so that the prism heights $z_{2}-z_{1}$ represent the MOLA residual elevations. The values $\left(x_{1}, y_{1}, x_{2}, y_{2}\right)$ are computed from the planetocentric coordinates of the computation point $(\varphi, \lambda)_{P}$ and of the prism $(\varphi, \lambda)_{Q}$ under evaluation using a simple spherical projection (after Forsberg, 1984)

$$
\begin{aligned}
& x_{1}=R \frac{2 \pi}{360}\left(\lambda_{Q}-\lambda_{P}-\frac{\Delta \lambda}{2}\right) \cos \varphi_{P} \\
& x_{2}=R \frac{2 \pi}{360}\left(\lambda_{Q}-\lambda_{P}+\frac{\Delta \lambda}{2}\right) \cos \varphi_{P} \\
& y_{1}=R \frac{2 \pi}{360}\left(\varphi_{Q}-\varphi_{P}-\frac{\Delta \varphi}{2}\right) \\
& y_{2}=R \frac{2 \pi}{360}\left(\varphi_{Q}-\varphi_{P}+\frac{\Delta \varphi}{2}\right)
\end{aligned}
$$

with $R=3389500 \mathrm{~km}$ as the mean radius of the Mars, $\Delta \lambda$ and $\Delta \varphi$ define the horizontal dimensions of the prism. A vertical shift of the prisms as a function of the distance between prism and computation point is applied to account for the effect of planetary curvature (cf. Forsberg, 1984, p. 111). The summation of gravity effects is performed over $k$ prisms within some radius (here $400 \mathrm{~km}$ ) around the computation point beyond which the addition of mass elements makes no significant difference (see Forsberg, 1984; Hirt, 2010).

The term $h c$ is termed the harmonic correction and applied to gravity disturbances $\delta g^{\text {MRTM }}$ as a correction for computation points located inside the residual topography (i.e., $z_{P}^{M R T M}<0$ ), cf. Forsberg and Tscherning (1981) 


$$
h c=\left\{\begin{array}{cc}
0 \quad, & z_{P}^{R T M} \geq 0 \\
4 \pi G \rho z_{P}^{R T M}, & z_{P}^{R T M}<0
\end{array}\right\}
$$

The harmonic correction is required because the gravity potential is non-harmonic inside the gravitating masses. Equation (24) solves this problem by condensing the residual masses in a “mass-plane just below the station” (Forsberg and Tscherning, 1981, p. 7846).

For computation points at high latitudes $\left(\left|\varphi_{P}\right| \geq 70^{\circ}\right)$, we rotated the RTM grids and computation points towards the equator, allowing us to conveniently circumvent computational issues at high latitudes (e.g., use of planar coordinates, increasing number of prisms due to meridian convergence and selection of prisms within a given radius). The Newtonian forward-modelling procedure, was carried out over 25.92 million points covering the entire surface of Mars.

\section{A5 MGM2011 functionals}

MGM2011 functionals are the sum of MRO110B2 and the forward-modelled MRTM85 components

$$
\begin{aligned}
& \delta g^{M G M 2011}=\delta g^{M R O}+\delta g^{M R T M} \\
& \xi^{M G M 2011}=\xi^{M R O}+\xi^{M R T M} \\
& \eta^{M G M 2011}=\eta^{M R O}+\eta^{M R T M}
\end{aligned}
$$

and MGM2011 surface gravity accelerations are the sum of normal gravity $\gamma(h, \phi)$ and MGM2011 gravity disturbances

$$
g^{M G M 2011}=\delta g^{M G M 2011}+\gamma(h, \phi) \text {. }
$$

\section{References}

Ardalan, A.A., Karimi, R., Grafarend, E.W., 2009. A new reference equipotential surface, and reference ellipsoid for the planet Mars, Earth Moon Planet, 106, 1-13.

Claessens, S.J., 2006. Solutions to ellipsoidal boundary value problems for gravity field modelling, $\mathrm{PhD}$ thesis, Department of Spatial Sciences, Curtin University of Technology, Perth, Australia.

Forsberg, R., 1984. A study of terrain reductions, density anomalies and geophysical inversion methods in gravity field modelling, Report 355, Department of Geodetic Science and Surveying, Ohio State University, Columbus. 
Forsberg, R., Tscherning, C.C., 1981. The use of height data in gravity field approximation by collocation, J. Geophys. Res, 86(B9), 7843-7854.

Goossens, S., Matsumoto, K., Liu, Q., Kikuchi, F., Sato, K., Hanada, H., Ishihara, Y., Noda, H., Kawano, N., Namiki, N., Iwata, T., Lemoine, F.G., Rowlands, D.D., Harada, Y., Chen, M., 2011. Lunar gravity field determination using SELENE same-beam differential VLBI tracking data, J Geod, 85, 205-228.

Grejner-Brzezinska, D.A., Wang, J., 1998. Gravity modeling for high-accuracy GPS/INS integration, Navigation, 45, 3, 209-220.

Gwinner, K., Scholten, F., Preusker, F., Elgner, S., Roatsch, T., Spiegel, M., Schmidt, R., Oberst, J., Jaumann, R., Heipke, C., 2010. Topography of Mars from global mapping by HRSC high resolution digital terrain models and orthoimages: Characteristics and performance, Earth Plan Sci Lett, 294, 506-519.

Holmes, S.A., Pavlis N.K., 2008. Spherical harmonic synthesis software harmonic_synth. http://earth-info.nga.mil/GandG/wgs84/gravitymod/new_egm

Hirt, C., 2010. Prediction of vertical deflections from high-degree spherical harmonic synthesis and residual terrain model data, J Geod, 84, 179-190.

Hirt, C., Featherstone, W.E., Marti, U., 2010. Combining EGM2008 and SRTM/ DTM2006.0 residual terrain model data to improve quasigeoid computations in mountainous areas devoid of gravity data, J Geod, 84, 557-567.

Hirt, C., Featherstone, W.E., 2011. A 1.5km-resolution gravity field model of the Moon. Earth Plan. Sci. Lett., submitted for publication.

Hirt, C., Gruber T., Featherstone, W.E, 2011a. Evaluation of the first GOCE static gravity field models using terrestrial gravity, vertical deflections and EGM2008 quasigeoid heights, J Geod, 85(10), 723-740, DOI: 10.1007/s00190-011-0482-y.

Hirt, C., Claessens S.J., Kuhn M., Featherstone W.E., 2011b. Implicit validation of Mars Gravity Model 2011 using a replication experiment on Earth, Stud. Geoph. Geod, submitted for publication.

Jekeli, C., 2001. Intertial navigation systems with geodetic applications, de Gruyter, Berlin.

Konopliv, A.S., Asmar, S.W., Folkner, W.M., Karatekin, Ö., Nunes, D.C., Smrekar, S.E., Yoder, C.F., Zuber, M.T., 2011. Mars high resolution gravity fields from MRO, Mars seasonal gravity, and other dynamical parameters, Icarus, 211, 401-428.

Lemoine, F.G., Smith D.E., Rowland, D.D., Zuber, M.T., Neumann, G.A., Chinn, D.S., Pavlis, D.E., 2001. An improved solution of the gravity field of Mars (GMM-2B) from Mars Global Surveyor, J. Geophys. Res., 106(E10), 23359-23376. 
Marty, J.C., Balmino, G., Duron, J., Rosenblatt, P., LeMaistre, S., Rivoldini, A., Dehant, V., Van Hoolst T., 2009. Martian gravity field model and its time variations from MGS and Odyssey data, Planet. Space Sci., 57, 350-363.

McGovern, P.J., Solomon, S.C., Smith, D.E., Zuber, M.T., Simons, M., Wieczorek M.A., Phillips R.J., Neumann G.A., Aharonson O., Head, J.W., 2002. Localized gravity/topography admittance and correlation spectra on Mars: Implications for regional and global evolution, J. Geophys. Res., 107(E12), 5136, doi: 10.1029/2002JE001854.

Moritz, H., 1980. Geodetic Reference System 1980, J Geod, 54(3), 395-405.

Moritz, H., 2000. Geodetic Reference System 1980, J. Geod, 74(1), 128-133.

Nagy, D., Papp, G., Benedek J., 2000. The gravitational potential and its derivatives for the prism, J. Geod 74, 552-560, Erratum in J. Geod 76, 475.

Neumann, G. A., Zuber, M.T., Wieczorek, M.A., McGovern P.J., Lemoine F.G., Smith D.E., 2004. Crustal structure of Mars from gravity and topography, J. Geophys. Res., 109, E08002, doi: 10.1029/2004JE002262.

Pavlis, N.K., Factor, J.K., Holmes, S.A., 2007. Terrain-related gravimetric quantities computed for the next EGM, Proceed. 1st Intern. Symp. Intern. Gravity Field Service, Istanbul, pp 318-323.

Smith, D.A., 1998. There is no such thing as "The” EGM96 geoid: Subtle points on the use of a global geopotential model, International Geoid Service Bulletin 8, pp 17-28.

Smith, D.E., Zuber, M.T., Frey, H.V., et al., 2001. Mars Orbiter Laser Altimeter (MOLA): experiment summary after the first year of global mapping of Mars, J. Geophys. Res. 106 (E10), 23689-23722.

Seidelmann, P.K., Abalakin, V.K., Bursa, M., Davies, M. E., De Bergh, C., Lieske, J. H., Oberst, J., Simon, J. L., Standish, E. M., Stooke P., Thomas P. C., 2002. Report of the IAU/IAG working group on cartographic coordinates and rotational elements of the planets and Satellites: 2000. Celestial Mechanics and Dynamical Astronomy, 82(1), 83110. DOI: 10.1023/A:1013939327465

Torge, W., 2001. Geodesy $3^{\text {rd }}$ ed., de Gruyter, Berlin.

Watts, A.B., 2001. Isostasy and Flexure of the Lithosphere, Cambridge University Press.

Wieczorek, M.A., Zuber, M.T., 2004. Thickness of the Martian crust: Improved constraints from geoid-to-topography ratios, J. Geophys. Res., 109, E01009.

Wieczorek, M.A., 2007. Gravity and topography of the terrestrial planets. In: Treatise on Geophysics, vol. 10. Elsevier-Pergamon, Oxford, pp. 165-206. 
Table 1 Defining and derived constants of the Mars Geodetic Reference System MGRS

\begin{tabular}{ll}
\hline Defining constants (exact) & Value \\
\hline semimajor axis $a$ & $3395428 \mathrm{~m}$ \\
semiminor axis $b$ & $3377678 \mathrm{~m}$ \\
gravitational constant times mass GM & $4.2828372 \times 10^{13} \mathrm{~m}^{3} \mathrm{~s}^{-2}$ \\
Rotational angular velocity $\omega$ & $7.0882181 \times 10^{-05} \mathrm{rad} \mathrm{s}^{-1}$ \\
\hline
\end{tabular}

\section{Derived geometric constants}

Geometric flattening $f$

Reciprocal flattening $1 / f$

First eccentricity squared $e^{2}$
$5.227617843759 \times 10^{-3}$

$1.912917183099 \times 10^{2}$

$1.042790769920 \times 10^{-2}$

\section{Derived physical constants}

Normal gravity at equator $\gamma_{a}$

$3.708754657884 \mathrm{~m} \mathrm{~s}^{-2}$

Normal gravity at pole $\gamma_{b}$

$3.731907392737 \mathrm{~m} \mathrm{~s}^{-2}$

Geodetic parameter $m$

$4.568250121143 \times 10^{-3}$

Dynamic form factor $J_{2}$

$1.955484200411 \times 10^{-3}$

Coefficient $C_{2,0}$

$-8.745191202090 \times 10^{-4}$

Coefficient $C_{4,0}$

$2.719280814521 \times 10^{-6}$

Coefficient $C_{6,0}$

$-1.217355067691 \times 10^{-8}$

Coefficient $C_{8,0}$

$6.522150395570 \times 10^{-11}$

Coefficient $C_{10,0}$

$-3.884319022342 \times 10^{-13}$

Note: Parameter $m$ is the ratio of the centrifugal acceleration to normal gravity at equator. Coefficients $C_{2,0}$ to $C_{10,0}$ are fully-normalized spherical harmonic coefficients of the MGRS.

Table 2 Descriptive statistics of MGM2011 surface gravity and MGM2011 free-air anomalies and of the three input components ${ }^{\mathrm{a}}$. Units in $10^{-5} \mathrm{~ms}^{-2}=1 \mathrm{mGal}$.

\begin{tabular}{lllll}
\hline Field/component & Min & Max & Mean & STD $^{\mathbf{b}}$ \\
\hline MGM2011 surface gravity acceleration & 368381 & 374259 & 372076 & 1115 \\
MGM2011 free-air gravity disturbances & -1107 & 2905 & 4 & 161 \\
\hline Normal gravity field & 365966 & 374343 & 372072 & 1148 \\
MRO110B2 gravity (band 2 to 85) & -774 & 2951 & 5 & 155 \\
MRTM85 Newtonian gravity & -956 & 705 & -1 & 41 \\
\hline
\end{tabular}

${ }^{\mathrm{a}}$ Input components are MRTM85, MRO110B (band 2 to 85) and normal gravity.

${ }^{\mathrm{b}} \mathrm{STD}=$ Standard deviation. 
Table 3 Descriptive statistics of MRTM85 geoid and MGM2011 vertical deflections (DoV) and their input components ${ }^{\mathrm{a}}$

\begin{tabular}{|c|c|c|c|c|}
\hline Field/component & Min & Max & Mean & STD \\
\hline MRTM85 geoid [m] & -39.0 & 39.9 & 0.0 & 2.3 \\
\hline MGM2011 N-S DoV["] & -896 & 942 & 5 & 52 \\
\hline MRO110B2 N-S DoV ["] & -907 & 696 & 5 & 49 \\
\hline MRTM85 N-S DoV["] & -399 & 382 & -0 & 17 \\
\hline MGM2011 E-W DoV["] & -1074 & 829 & 0 & 63 \\
\hline MRO110B2 E-W DoV["] & -837 & 739 & 0 & 62 \\
\hline MRTM85 E-W DoV["] & -338 & 285 & 0 & 15 \\
\hline MGM2011 total DoV ["] & 0 & 1074 & 63 & 52 \\
\hline
\end{tabular}

